\title{
Simulation of the process of movement of bulk material in a vibrating conveyor-mixer
}

\author{
Tatiana Tupolskikh ${ }^{1, *}$, Natalia Gucheva ${ }^{1}$, and Oleg Kirishiev ${ }^{1}$ \\ ${ }^{1}$ Don State Technical University, Gagarin square 1, Rostov-on-Don, 344000, Russia
}

\begin{abstract}
The article considers the hypothesis that one of the factors affecting the processes occurring in the tray is the nature of the vibration effect: frequency, amplitude, direction of oscillation. There arises the task of constructing a model of the motion of particles of granular medium in the tray of a vibrating conveyor-mixer. To solve the problem it is necessary to use two coordinate systems: movable and fixed.
\end{abstract}

The working body of the vibrating conveyor-mixer is an inclined tray of a given section that performs oscillations in the vertical plane [1,2]. It is established that in the process of vibrating action on a product placed in a tray, internal flows arise in it, the result of which is mixing. The presence of different products in the tray leads to mixing.

The initial hypothesis is that one of the factors influencing the processes occurring in the tray is the nature of the vibration effect: frequency, amplitude, direction of oscillation. There arises the task of constructing a model of the motion of particles of granular medium in the chute of a vibrating conveyor-mixer.

Figure 1 shows a diagram for solving the modeling problem - the cross section of the tray in the form of an arc of a circle.

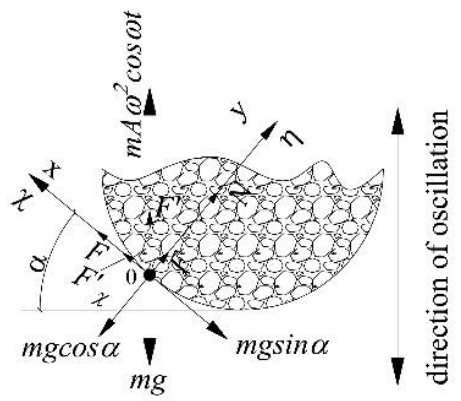

Fig. 1. The coordinate system and the scheme of the forces acting on the particle in the free-flowing layer.

\footnotetext{
Corresponding author: dstu-article@yandex.ru
} 
The cross section is symmetrical with respect to the longitudinal axis of the tray, so we will consider the movement of the layer on the left side of the tray, assuming that the right side of the bottom of the mixer of motion will be a mirror image of the motion on the left.

To solve the problem it is necessary to use two coordinate systems: movable and fixed.

The movable rectangular coordinate system $X O Y$ is connected to the bottom of the mixer. The origin at the point $O$ of the mixer bottom, angle of the $O X$ axis to the horizon $\alpha$. Fixed coordinate system $\chi O_{1} \eta$ is related to the material particle of the product $m$, located on the surface of the tray, and parallel to the movable coordinate system.

To solve the problem, we make the following assumptions:

1. at the initial instant of time the origin of coordinates $O$ and $O_{1}$ coincide;

2. the direction of oscillations forms with a horizontal plane a given constant angle equal to $\pi / 2$;

3. a material particle of mass $m$ is an elementary volume of granular medium with its properties;

4. the force of resistance of a granular medium to the motion of a particle $F^{\prime}$ is proportional to the velocity of motion of the particle and is the same in all directions;

5. The friction coefficient of the particle sliding along the surface of the bottom of the mixer $f$ is a constant.

The motion of the bottom will be harmonic, determined by a system of equations in a fixed coordinate system:

$$
\left\{\begin{array}{l}
\chi=A \cos \omega t \sin \alpha \\
\eta=A \cos \omega t \cos \alpha
\end{array}\right.
$$

where $\omega$ - circular oscillation frequency; $A$ - amplitude of oscillation.

The first derivative (velocity) and the second derivative (acceleration) will have the form:

$$
\left\{\begin{array} { l } 
{ \frac { d \chi } { d t } = - A \omega \operatorname { s i n } \omega t \operatorname { s i n } \alpha } \\
{ \frac { d \eta } { d t } = - A \omega \operatorname { s i n } \omega t \operatorname { c o s } \alpha }
\end{array} \left\{\begin{array}{l}
\frac{d^{2} \chi}{d t^{2}}=-A \omega^{2} \cos \omega t \sin \alpha \\
\frac{d^{2} \eta}{d t^{2}}=-A \omega^{2} \cos \omega t \cos \alpha
\end{array}\right.\right.
$$

The influence of the product layer on the particle $\mathrm{m}$ is determined by the additional force $F^{\prime}$ resistance to movement, proportional to the velocity of the particle and identical in all directions. We define this force as follows:

$$
\text { Axis } 0 \chi \quad F_{\chi}^{\prime}=k^{\prime} \dot{\chi} \text {, and axis } 0 \eta \quad F_{\chi}^{\prime}=k^{\prime} \dot{\eta}
$$

where $-k^{\prime}$ coefficient of proportionality.

Since it is assumed that the coefficient of friction $f$ is a constant, the differential equations of motion of the particle when vibrating the layer of bulk material will take the form:

$$
\left\{\begin{array}{c}
m \ddot{\chi}=f N+k^{\prime} \dot{\chi}-m g \sin \alpha \\
m \ddot{\eta}=N+k^{\prime} \dot{\eta}-m g \cos \alpha
\end{array}\right.
$$

Of interest is the motion of a particle in the mobile $X O Y$ coordinate system, which has the form, taking into account the portable movement of the bottom of the mixer:

$$
\left\{\frac{m \ddot{X}=f N+k^{\prime} \dot{\chi}-m g \sin \alpha+m A \omega^{2} \cos \omega t \sin \alpha}{m \ddot{Y}=N+k^{\prime} \dot{\eta}-m g \cos \alpha+m A \omega^{2} \cos \omega t \cos \alpha}\right.
$$


The direction of the frictional force depends on the direction of motion of the particle and can be defined as:

$$
F=\left\{\frac{-f N,}{f N,} \text { if } \frac{\dot{X}>0}{\dot{X}<0}\right.
$$

It is known that in the vibrational displacement of a particle there are different modes of motion: motion along with the plane and motion outside the plane. When moving along with the plane, the particle can be relatively fixed, move forward or backward [3, 4]. With vertical vibrations, the particle (layer) can move together with the plane, and move vertically outside the plane. To solve the equations of motion of a particle, we find the moments of a change in the form of the particle motion. The motion of the particles outside the plane or with separation from the bottom is of interest for the mixing process, since in this case, as experiments have shown [5,6], there is an intensive mixing of the particulate material with air flowing between the particles and relative displacement of the particles.

Let us find the moment of transition of the motion of a particle with the plane of the bottom to the motion of a particle outside the plane of the bottom, otherwise, the moment of separation $t_{0}$ of the particle from the bottom

. If we multiply $t_{0}$ by the circular oscillation frequency $\omega$, we obtain the angle of separation of the layer from the bottom $\omega t_{0}$.

It is obvious that at the moment of separation $t_{0}$ of the particle from the plane of the bottom, the normal pressure $N=0$.

Normal pressure is present in the second equation of the system of equations (4). Acceleration of the bottom at the time of separation $t_{0}$ should be $\ddot{Y}=-g$. In this case, the equation takes the form:

$$
m(-g)=N+k^{\prime} A \omega \sin \omega t_{0} \cos \alpha-m g \cos \alpha+m A \omega^{2} \cos \omega t_{0} \cos \alpha
$$

After the transformations, taking into account that $N=0$, we obtain:

$$
(-g)=-k A \omega \sin \omega t_{0} \cos \alpha-g \cos \alpha+A \omega^{2} \cos \omega t_{0} \cos \alpha
$$

where $k=\frac{k \prime}{m}$ - coefficient of resistance of granular medium to particle motion.

Further transformations lead to an expression that determines the angle of separation $\omega t_{0}$ of the layer of granular material under vibration action:

$$
\omega t_{0}=\arccos \left[\frac{g}{A \omega^{2}}\left(\frac{\cos \alpha-1}{\cos \alpha}\right) \cdot \operatorname{arctg} B\right]
$$

where $B=\frac{k}{\omega}$.

The resistance coefficient $k$ represents the integral effect of the layer on a particle $m$ and can be found by an experimental method, since it depends on the physico-mechanical properties of the material being transported.

Let us construct a diagram of the change in the angle of separation $\omega t_{0}$ from the angle of inclination $\alpha$ of the profile of the bottom of the mixer (Figure 2) to the horizon according to equation (7). 


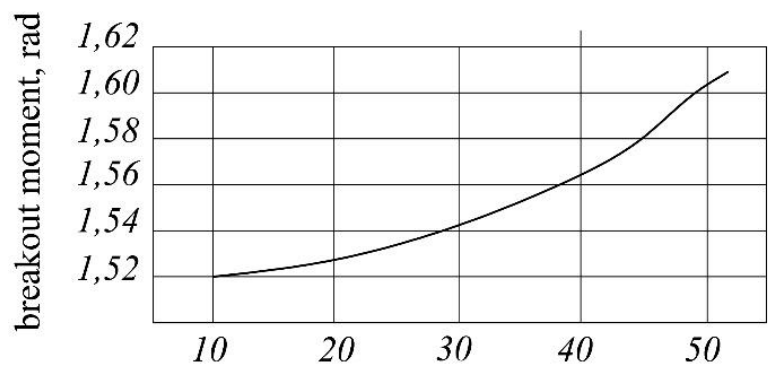

The angle of inclination of the bottom, $\alpha^{0}$

Fig. 2. Change in the angle of separation of the layer $\omega t_{0}$ from the angle of inclination $\alpha$ of the bottom profile. Coefficient of resistance $k=5 \mathrm{~s}^{-1}$, oscillation frequency $\omega=161 \mathrm{~s}^{-1}$, amplitude of oscillation $\mathrm{A}=0,0025 \mathrm{~m}$.

\section{Summary}

It follows from the diagram that the separation moment $\omega t_{0}$ depends on the position of the particle at the bottom of the tray - the particles break away from the bottom of the tray not simultaneously. The smaller the angle, the earlier the particle separates. This circumstance leads to a displacement of the particles within the layer in such a way that the circular motion of the particles in the cross section. Two vortices are formed, rotating in the opposite direction. Such a motion of the particles of the layer leads to mixing of the particles. In the case of several ingredients in the layer, the fact described is the cause of mixing.

Figure 2 shows that the moment of separation of the particles of the layer lags with respect to the initial moment at the bottom point of the bottom as the angle $\alpha$ of the bottom profile increases. As a result, the particles located above descend downward before the particles below. This creates two vortices in the cross section of the flow rotating in opposite directions.

\section{References}

1. V.I. Pakhomov, N.V. Gucheva, D.V. Rudoi, SPRSM, Vibro-mixer for the preparation of small-sized biologically active feed additives, 11, 577-580 (2018)

2. N.V. Gucheva, V.D. Ilyichenko, E.V. Revyakin, SPRSM, Vibratory mixer of dry ingredients of mixed fodders, 13, 156-158 (2010)

3. I.I. Blechman, G.Y. Dzhanelidze, Vibrational movement, 410 (1964)

4. N.V. Gucheva, Bulletin of the DSTU, Investigation of vibrational mixing of bulk granular materials, 9, 151-163 (2009)

5. N.V. Gucheva, V.D. Ilyichenko, T.I. Tupolskikh, ИТНО-2014, Experimental studies of the process of vibrational mixing in a mixer with a profile bottom, 4 - 7 September 2014, 238-243 (2014)

6. T.I. Tupolskikh, V.D. Ilyichenko, Vestnik DGTU, Investigation of the technological process of operation of a vibrating feeder with a hopper, 1 (3), 56-64 (2001) 